\title{
Sonographic Measurement of Optic Nerve Sheath Diameter: How Steep is the Learning Curve for a Novice Operator?
}

\author{
Gentle Sunder Shrestha, Binayak Upadhyay', Aanchal Shahi', Jaya Ram K. C', Pankaj Joshi, Bishesh Sharma Poudyal² \\ Department of Anaesthesiology, Institute of Medicine, Tribhuvan University Teaching Hospital, ${ }^{2}$ Department of Clinical Hematology and Bone Marrow Transplant Unit, \\ Civil Service Hospital, Kathmandu, 'Department of Critical Care, Alka Hospital Private Limited, Jawalakhel, Lalitpur, Nepal
}

\section{Abstract}

Background: Transbulbar sonography for measuring optic nerve sheath diameter (ONSD) is noninvasive bedside technique for detection of raised intracranial pressure. This study aims to evaluate the number of sonographic evaluations required for a novice operator to learn proper measurement of ONSD after a formal training and supervised scanning session. Methods: Three novice operators and one expert operator measured ONSD of 27 healthy volunteers using linear array transducer HFL38x (frequency range of 6-13 MHz) (MicroMaxx®; SonoSite, USA). In each eye, ONSD was measured three times by each observer and mean value was determined. Correlation coefficient was calculated between the novice and the experienced operator and in between the novice operators. Number of scans, after which the significant correlation developed between novice and the experienced operator and between the novice operators, was analyzed. Results: Cronbach's alpha was tested to evaluate the reliability of the values obtained from intra- and interobservations. For all 27 cases, the value of Cronbach's alpha was high: 0.80-0.99 for the right eye and 0.69-0.97 for the left eye. The mean interobserver variations were plotted on a graph which fluctuated largely in the first 17 cases but oscillated around $0.5-0.30$ in the last 10 cases. Conclusion: Learning curve for novice operators to measure ONSD is steep and they can be taught to measure ONSD in healthy volunteers by sonography in supervised clinical session with an acceptable clinical precision and accuracy comparable to an experienced operator.

Keywords: Learning curve, optic nerve sheath diameter, ultrasonography

\section{INTRODUCTION}

Raised intracranial pressure (ICP) is the most significant cause of secondary brain injury and leads to poor outcome. If left untreated, it can lead to brain ischemia and brain stem herniation. Invasive intracranial devices are the gold standard for measuring ICP. However, it is not always feasible and not free of complications. Ultrasonography is widely available bedside noninvasive technique. Optic nerve sheath diameter (ONSD) measurement can be used as a noninvasive surrogate of raised ICP and has a good diagnostic accuracy. ${ }^{[1,2]}$ Proper and adequate training is crucial to improve diagnostic accuracy and to avoid improper management. Previous studies report good intra- and interobserver reliability between the experts and between expert and novice after training and practice. ${ }^{[3,4]}$ However, well-designed studies addressing the learning curve are lacking.

This study aims to evaluate the number of sonographic evaluations required for a novice operator to learn proper

\begin{tabular}{|l|l|}
\hline \multicolumn{3}{c|}{ Access this article online } \\
\hline Quick Response Code: & Website: \\
& www.ijccm.org \\
\hline
\end{tabular}

measurement of ONSD after a formal training and supervised scanning session. It aims to evaluate the interobserver reliability and variability of ONSD measurement between the novice operators and the experienced operator. The study also evaluates the interobserver reliability and variability of ONSD measurement between the novice operators.

\section{Materials and Methods}

Ethical approval was obtained from the Institution Review Board and Ethical Committee. A cross-sectional, observational study was performed. The operators with no previous experience of ONSD measurement was designated as novice

Address for correspondence: Dr. Gentle Sunder Shrestha, Department of Anaesthesiology, Tribhuvan University Teaching Hospital, Institute of Medicine, Maharajgunj, Kathmandu, Nepal. E-mail: gentlesunder@hotmail.com

This is an open access journal, and articles are distributed under the terms of the Creative Commons Attribution-NonCommercial-ShareAlike 4.0 License, which allows others to remix, tweak, and build upon the work non-commercially, as long as appropriate credit is given and the new creations are licensed under the identical terms.

For reprints contact: reprints@medknow.com

How to cite this article: Shrestha GS, Upadhyay B, Shahi A, Jaya Ram KC, Joshi P, Poudyal BS. Sonographic measurement of optic nerve sheath diameter. How steep is the learning curve for a novice operator? Indian J Crit Care Med 2018;22:646-9. 
operators. The operator with $>200$ previous experience of ONSD measurement was designated as an experienced operator (Operator 1). Three novice operators (operators 2-4) were trained in a $2 \mathrm{~h}$ practical session consisting of $30-\mathrm{min}$ lecture describing orbital anatomy and technique for ONSD measurement. It was followed by real-time demonstration of ONSD measurement by the experienced operator on the healthy volunteers. Novices were then supervised by the experienced operator to perform measurement of ONSD on volunteers. Each novice acquired at least 10 images of sufficient quality and with a proper technique during the session of supervised scans.

Written informed consent was obtained from the participating volunteer. Adult healthy volunteers of either sex, 16 years and above, and without know ocular pathology were enrolled in the study. All the measurements were made using linear array transducer HFL38x (frequency range of 6-13 $\mathrm{MHz}$ ) (MicroMaxx ${ }^{\circledR}$; SonoSite, USA). The mechanical index was reduced to 0.2 and the thermal index to 0.0 to avoid biomechanical side effects. ${ }^{[5]}$ Patients were kept lying supine with the upper body elevated at $0^{\circ}-30^{\circ}$ and the head in neutral position. Patients were asked to close the eyes and to keep the eyes fixed in a neutral position. Ultrasound gel was applied to the outside of each upper eyelid. Transducer was placed horizontally. Electronic caliper was used to mark the point $3 \mathrm{~mm}$ behind the globe. ONSD was measured at that depth at right angle to the optic nerve. The widest diameter visible was recorded. Three readings were obtained from each eye. The readings were recorded by a person not involved in the study. The observers were blinded to the readings obtained. At the $95 \%$ confidence level and $80 \%$ power, to determine the average difference of $0.13 \mathrm{~mm}$, the required sample size was 27 cases.

A total of 27 healthy volunteers were scanned for ONSD measurement. The measurements obtained by the novice operator in each eye were compared with that obtained by the experienced operator in every case. Correlation coefficient was calculated between the novice and the experienced operator and in between the novice operators. Number of scans, after which the significant correlation develops between novice and the experienced operator and between the novice operators, was analyzed. Number of scans after which inter- and intraobserver variability gets minimum and plateaus, was analyzed.

\section{RESULTS}

Readings on ONSD were conducted among 27 individuals (known as volunteers) aged from 17 to 46 years (mean age 27.41 years). Of them, $19(70.40 \%)$ were female and the rest $8(29.60 \%)$ were male.

The overall mean of ONSD for the right and left eyes was around 3.40 and $3.57 \mathrm{~mm}$, respectively. The mean value of ONSD for the right eye ranged from a minimum of $2.43 \mathrm{~mm}$ to a maximum of $4.30 \mathrm{~mm}$ while in case of the left eye, it ranged from $2.27 \mathrm{~mm}$ to $4.47 \mathrm{~mm}$ [Table 1].

The difference in measurement of ONSD (mean values) between the operators was calculated. The difference between experienced and novice operators was between $0.27 \mathrm{~mm}$ and $0.31 \mathrm{~mm}$ for the right eye and was between $0.18 \mathrm{~mm}$ and $0.24 \mathrm{~mm}$ for the left eye. The values of standard deviation were higher than the corresponding mean except for the difference between average values of observers 1 and 3 for the right eye and 1 and 4 for both the eyes. It implies there was a larger variability in the readings of different observers compared to the mean values. The difference between the average values of the observations between the experienced and novice operators ranged from the minimum of $0 \mathrm{~mm}$ for both eyes to maximum of $1.13 \mathrm{~mm}$ and $1.27 \mathrm{~mm}$, respectively, for the right and left eyes. Interestingly, for the initial 17 cases, the variability between the average readings of different observers was higher $(0.22-0.45 \mathrm{~mm})$ compared to all 27 cases. However, the readings were more uniformly distributed for the last 10 cases (variability of $0.09-0.13 \mathrm{~mm}$ ) [Table 2].

Cronbach's alpha test was conducted to evaluate the reliability and internal consistency of the values obtained from intra- and interobservations. For all 27 cases, the value of Cronbach's alpha was found to be $0.80-0.99$ for the right eye and $0.69-0.97$ for the left eye. The same for the initial 17 cases ranged from 0.40 to 0.98 for the right eye and $0.55-0.98$ for the left eye. While for the last 10 cases, alpha was $0.93-0.99$ for the right eye and $0.74-0.91$ for the left eye. The acceptable range for Cronbach's alpha theoretically is from 0.70 to 0.90 . The value lower than 0.70 indicates poor reliability and internal consistency while the value higher than 0.90 suggests redundancy. The mean values of Cronbach's alpha obtained from comparison between operator 1 and 2 were 0.82 for the right eye and 0.72 for the left eye; between 1 and 3 were 0.71 for the right eye and 0.81 for the left eye and between 1 and 4 were 0.84 for the right eye and 0.80 for the left eye. The value

\begin{tabular}{|c|c|c|c|c|c|c|c|c|}
\hline \multirow[t]{2}{*}{ Operators } & \multicolumn{2}{|c|}{ Mean (mm) } & \multicolumn{2}{|c|}{ SD (mm) } & \multicolumn{2}{|c|}{ Minimum (mm) } & \multicolumn{2}{|c|}{ Maximum (mm) } \\
\hline & Right eye & Left eye & Right eye & Left eye & Right eye & Left eye & Right eye & Left eye \\
\hline 1 & 3.49 & 3.49 & 0.52 & 0.38 & 2.53 & 2.60 & 4.27 & 4.47 \\
\hline 2 & 3.42 & 3.40 & 0.54 & 0.40 & 2.43 & 2.27 & 4.30 & 4.37 \\
\hline 3 & 3.42 & 3.50 & 0.35 & 0.28 & 2.67 & 3.00 & 3.87 & 4.03 \\
\hline 4 & 3.57 & 3.53 & 0.42 & 0.31 & 2.60 & 2.93 & 4.13 & 4.13 \\
\hline
\end{tabular}




\begin{tabular}{|c|c|c|c|c|c|c|c|c|}
\hline \multirow{2}{*}{$\begin{array}{l}\text { Difference } \\
\text { between operators }\end{array}$} & \multicolumn{2}{|c|}{ Mean (mm) } & \multicolumn{2}{|c|}{ SD (mm) } & \multicolumn{2}{|c|}{ Minimum (mm) } & \multicolumn{2}{|c|}{ Maximum (mm) } \\
\hline & Right eye & Left eye & Right eye & Left eye & Right eye & Left eye & Right eye & Left eye \\
\hline \multicolumn{9}{|c|}{ All 27 cases } \\
\hline 1 and 2 & 0.27 & 0.23 & 0.30 & 0.29 & 0.00 & 0.00 & 1.13 & 1.27 \\
\hline 1 and 3 & 0.31 & 0.18 & 0.28 & 0.20 & 0.00 & 0.00 & 1.07 & 0.80 \\
\hline 1 and 4 & 0.27 & 0.24 & 0.23 & 0.15 & 0.00 & 0.00 & 0.83 & 0.50 \\
\hline \multicolumn{9}{|c|}{ For initial 17 cases } \\
\hline 1 and 2 & 0.38 & 0.32 & 0.33 & 0.34 & 0.03 & 0.03 & 1.13 & 1.27 \\
\hline 1 and 3 & 0.45 & 0.22 & 0.28 & 0.23 & 0.17 & 0.00 & 1.07 & 0.80 \\
\hline 1 and 4 & 0.35 & 0.30 & 0.25 & 0.13 & 0.00 & 0.07 & 0.83 & 0.50 \\
\hline \multicolumn{9}{|c|}{ Last 10 cases } \\
\hline 1 and 2 & 0.09 & 0.09 & 0.09 & 0.09 & 0.00 & 0.00 & 0.30 & 0.23 \\
\hline 1 and 3 & 0.09 & 0.11 & 0.09 & 0.10 & 0.00 & 0.00 & 0.30 & 0.23 \\
\hline 1 and 4 & 0.12 & 0.13 & 0.09 & 0.10 & 0.03 & 0.00 & 0.27 & 0.37 \\
\hline
\end{tabular}

between operators 2,3 , and 4 was 0.88 for the right eye and 0.80 for the left eye [Table 3].

For the right eye, the averages at $5^{\text {th }}, 50^{\text {th }}$, and $95^{\text {th }}$ percentiles fluctuated from 2.45 to $2.67 \mathrm{~mm}, 3.50$ to $3.63 \mathrm{~mm}$, and 3.87 to $4.29 \mathrm{~mm}$, respectively. For the left eye, the averages ranged from $2.53-3.00 \mathrm{~mm}, 3.33-3.57 \mathrm{~mm}$, and $3.99-4.25 \mathrm{~mm}$, respectively. Similar to the mean values for the initial 17 cases, the corresponding averages at $5^{\text {th }}, 50^{\text {th }}$, and $95^{\text {th }}$ percentiles were on the greater side depicting larger fluctuations.

Karl Pearson's correlation coefficient was determined to establish a relationship between measurements of various observers. The coefficient between observers 1 and 2 was found to be 0.697 for the right eye and 0.558 for the left eye, indicating a high positive correlation. Between observers 1 and 3 , the coefficient was 0.598 for the right eye and 0.709 for the left eye. Between observers 1 and 4, it was 0.749 for the right eye and 0.688 for the left eye. The correlation coefficients were higher in the last 10 cases $(0.975-0.980$ for the right eye and $0.788-0.846$ for the left eye) when compared to the initial 17 cases $(0.410-0.478$ for the right eye and $0.485-0.618$ for the left eye) [Table 4].

The difference in measurements obtained by operator 1 and 2 , 1 and 3 , and 1 and 4 were plotted in a graph. The lines of mean difference from cases 1 to 17 fluctuated largely with a highest peak of almost $1.20 \mathrm{~mm}$ to as low as $0.10 \mathrm{~mm}$. However, the lines of mean difference oscillated around $0.50-0.30 \mathrm{~mm}$ in cases 18-27 [Figure 1].

\section{DisCussion}

The use of ultrasound for measuring ONSD has been described in many medical literatures. ${ }^{[4,6]}$ Most of the studies show that this method is adequately sensitive and specific for diagnosing intracranial hypertension. Well-designed studies which measure the numbers of sonographic evaluation required for a novice operator to learn proper measurement of ONSD after
Table 3: Cronbach's alpha tested between the operators

\begin{tabular}{lcc}
\hline \multirow{2}{*}{$\begin{array}{l}\text { Operators and } \\
\text { corresponding reading }\end{array}$} & \multicolumn{2}{c}{ Cronbach's alpha } \\
\cline { 2 - 3 } & Right eye & Left eye \\
\hline Operator 1 versus 2 & 0.75 \\
Reading 1 & 0.74 & 0.55 \\
Reading 2 & 0.82 & 0.64 \\
Average & 0.82 & 0.72 \\
\hline & Operators 1 versus 3 \\
\hline Reading 1 & 0.58 \\
Reading 2 & 0.71 & 0.67 \\
Reading 3 & 0.65 & 0.82 \\
Average & 0.71 & 0.63 \\
\hline & Operators 1 versus 4 & 0.81 \\
\hline Reading 1 & 0.76 & 0.67 \\
Reading 2 & 0.64 & 0.57 \\
Reading 3 & 0.86 & 0.80 \\
Average & 0.84 & 0.80 \\
\hline & Operators 2, 3, and 4 \\
\hline Reading 1 & 0.79 & 0.70 \\
Reading 2 & 0.75 & 0.68 \\
Reading 3 & 0.80 & 0.65 \\
Average & 0.88 & 0.80 \\
\hline
\end{tabular}

formal training and supervised scanning session is lacking. As the use and availability of ultrasonography is on rise not only in ICU but also in primary level of health facilities, health-care personnels such as medical officers, intensivists, and even neurosurgeons can be taught to measure ONSD and establish this method as a valid noninvasive tool for measuring raised ICP. Developing proper training and competence in critical care ultrasound has been one of the major advances and goals of research agenda on critical care ultrasonography. ${ }^{[7]}$

The overall mean of ONSD and range of ONSD measurement: $2.43-4.30 \mathrm{~mm}$ for the right eye and $2.27-4.37 \mathrm{~mm}$ for the left 
Table 4: Karl Pearson's correlation coefficient between readings of experienced operator and novice operators

\begin{tabular}{lcc}
\hline Operators & \multicolumn{2}{c}{ Karl Pearson's correlation coefficient } \\
\cline { 2 - 3 } & Right eye & Left eye \\
\hline \multicolumn{3}{c}{ All 27 cases } \\
\hline 1 and 2 & 0.697 & 0.558 \\
1 and 3 & 0.598 & 0.709 \\
1 and 4 & 0.749 & 0.688 \\
\hline \multicolumn{3}{c}{ For initial 17 cases } \\
\hline 1 and 2 & 0.478 & 0.485 \\
1 and 3 & 0.410 & 0.618 \\
1 and 4 & 0.412 & 0.591 \\
\hline & Last 10 cases \\
\hline 1 and 2 & 0.975 & 0.846 \\
1 and 3 & 0.980 & 0.822 \\
and 4 & 0.980 & 0.788 \\
\hline
\end{tabular}

eye in our study is consistent with results from other studies like Ballantyne et al. ${ }^{\left[{ }^{[4]}\right.}$ Hansen et al. ${ }^{[8]}$ and Hansen and Helmke. ${ }^{[9]}$ In our study, after measuring ONSD in 17 cases, there was very little discrepancies between novice and expert operators for the last ten cases as variability between the average readings of different observers was less. These results are consistent with the results obtained from studies of Ballantyne et al. ${ }^{[4]}$ in which after 17 cases interobserver variation has decreased.

Karl Pearson's correlation coefficient was higher in the last ten cases compared to initial 17 cases and mean Cronbach's alpha was also higher for the last ten cases in our study which strengthens the concept that novice operators have steep learning curve for ONSD measurement.

Hence, the study shows that with careful training of the examination technique for ONSD measurements, novice operators can be trained and after measurement of ONSD in 17 cases, there is significant reduction in intra- and interobserver variation. Based on our study and the study by Ballentyne et al. ${ }^{[4]}$ the learning curve for ONSD measurement seems to be steeper as compared to the recommendations for acquiring competence in transthoracic echocardiography (100 scans), transesophageal echocardiography (35 scans), ${ }^{[7]}$ and for diagnostic lung ultrasonography (25 scans). ${ }^{[10]}$

This study has several limitations. It was a single-centered study and was conducted on the healthy volunteers. Studies designed to evaluate the intra- and interobserver variability of measurement of ONSD in patients with raised ICP would be prudent.

\section{Conclusion}

Learning curve for the novice operators to learn measurement of ONSD is steep. Well-designed standardized training and incorporation of recommendations in the curriculum of

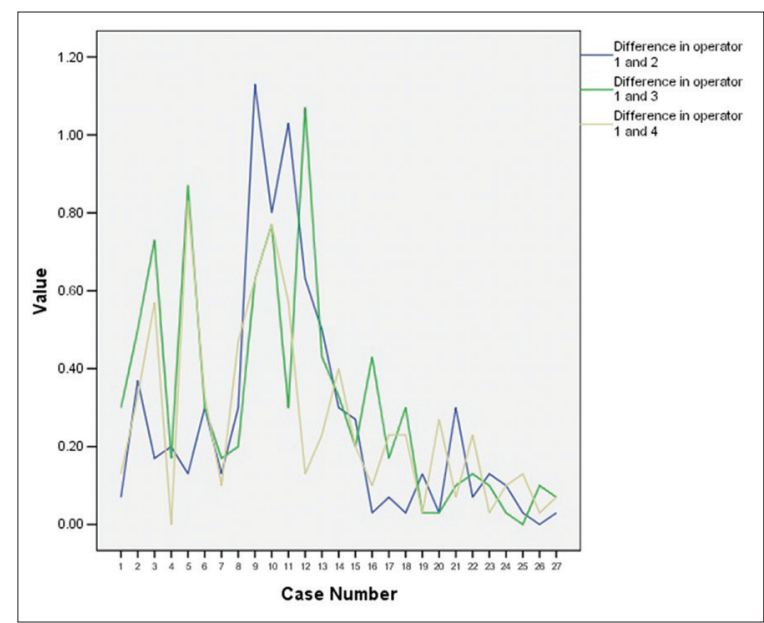

Figure 1: Difference in measurements obtained by operators

point-of-care ultrasonography can facilitate more effective use of this modality as a noninvasive surrogate for the detection of raised ICP.

\section{Financial support and sponsorship}

Nil.

\section{Conflicts of interest}

There are no conflicts of interest.

\section{RefEREnCES}

1. Dubourg J, Javouhey E, Geeraerts T, Messerer M, Kassai B. Ultrasonography of optic nerve sheath diameter for detection of raised intracranial pressure: A systematic review and meta-analysis. Intensive Care Med 2011;37:1059-68.

2. Shirodkar CG, Rao SM, Mutkule DP, Harde YR, Venkategowda PM, Mahesh MU, et al. Optic nerve sheath diameter as a marker for evaluation and prognostication of intracranial pressure in Indian patients: An observational study. Indian J Crit Care Med 2014;18:728-34.

3. Bäuerle J, Lochner P, Kaps M, Nedelmann M. Intra - And interobsever reliability of sonographic assessment of the optic nerve sheath diameter in healthy adults. J Neuroimaging 2012;22:42-5.

4. Ballantyne SA, O'Neill G, Hamilton R, Hollman AS. Observer variation in the sonographic measurement of optic nerve sheath diameter in normal adults. Eur J Ultrasound 2002;15:145-9.

5. Bäuerle J, Schuchardt F, Schroeder L, Egger K, Weigel M, Harloff A, et al. Reproducibility and accuracy of optic nerve sheath diameter assessment using ultrasound compared to magnetic resonance imaging. BMC Neurol 2013;13:187.

6. Soldatos T, Karakitsos D, Chatzimichail K, Papathanasiou M, Gouliamos A, Karabinis A, et al. Optic nerve sonography in the diagnostic evaluation of adult brain injury. Crit Care 2008;12:R67.

7. Mayo P, Arntfield R, Balik M, Kory P, Mathis G, Schmidt G, et al. The ICM research Agenda on critical care ultrasonography. Intensive Care Med 2017;43:1257-69.

8. Hansen HC, Helmke K, Kunze K. Optic nerve sheath enlargement in acute intracranial hypertension. Neuro Ophthalmol 1994;14:345-54.

9. Hansen HC, Helmke K. Validation of the optic nerve sheath response to changing cerebrospinal fluid pressure: Ultrasound findings during intrathecal infusion tests. J Neurosurg 1997;87:34-40.

10. Kristensen MS, Teoh WH, Graumann O, Laursen CB. Ultrasonography for clinical decision-making and intervention in airway management: From the mouth to the lungs and pleurae. Insights Imaging 2014;5:253-79. 